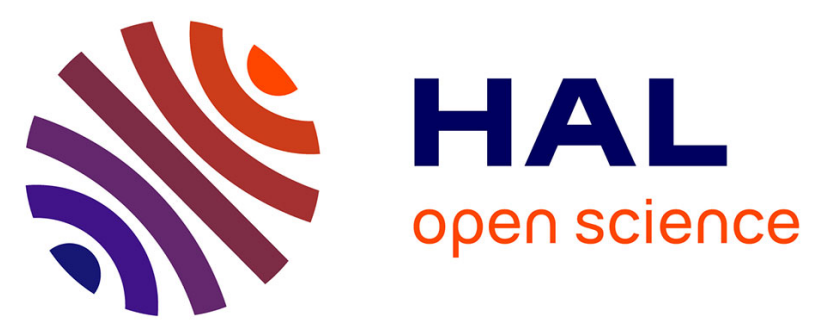

\title{
Anomalous Magnetic Field Dependence of the Thermodynamic Transition Line in the Isotropic Superconductor K,Ba BiO3
}

S. Blanchard, Thierry Klein, J. Marcus, I. Joumard, A. Sulpice, P. Szabo, P. Samuely, A. G. M. Jansen, C. Marcenat

\section{To cite this version:}

S. Blanchard, Thierry Klein, J. Marcus, I. Joumard, A. Sulpice, et al.. Anomalous Magnetic Field Dependence of the Thermodynamic Transition Line in the Isotropic Superconductor K,Ba BiO3. Physical Review Letters, 2002, 88, pp.177201. 10.1103/PhysRevLett.88.177201 . hal-00959104

\section{HAL Id: hal-00959104 https://hal.science/hal-00959104}

Submitted on 13 Mar 2014

HAL is a multi-disciplinary open access archive for the deposit and dissemination of scientific research documents, whether they are published or not. The documents may come from teaching and research institutions in France or abroad, or from public or private research centers.
L'archive ouverte pluridisciplinaire HAL, est destinée au dépôt et à la diffusion de documents scientifiques de niveau recherche, publiés ou non, émanant des établissements d'enseignement et de recherche français ou étrangers, des laboratoires publics ou privés. 


\title{
Anomalous Magnetic Field Dependence of the Thermodynamic Transition Line in the Isotropic Superconductor $(\mathrm{K}, \mathrm{Ba}) \mathrm{BiO}_{3}$
}

\author{
S. Blanchard, ${ }^{1}$ T. Klein,,${ }^{1,2}$ J. Marcus, ${ }^{1}$ I. Joumard,${ }^{1}$ A. Sulpice, ${ }^{3}$ P. Szabo, ${ }^{2,4}$ \\ P. Samuely, ${ }^{4}$ A. G. M. Jansen, ${ }^{5}$ and C. Marcenat ${ }^{6}$ \\ ${ }^{1}$ Laboratoire d'Etudes des Propriétés Electroniques des Solides, Centre National de la Recherche Scientifique, \\ BP 166, F-38042 Grenoble Cedex 9,France \\ ${ }^{2}$ University Joseph Fourier, BP 53, F-38041 Grenoble Cedex 9, France \\ ${ }^{3}$ Centre de Recherche sur les Très Basses Températures, Centre National de la Recherche Scientifique, \\ BP 166, 38042 Grenoble Cedex 9, France \\ ${ }^{4}$ Institute of Experimental Physics, Slovak Academy of Sciences, SK-04353 Košice, Slovakia \\ ${ }^{5}$ Grenoble High Magnetic Field Laboratory, Max-Planck-Institut für Festkörperforschung and \\ Centre National de la Recherche Scientifique, BP 166, F-38042 Grenoble Cedex 9, France \\ ${ }^{6}$ Commissariat à l'Energie Atomique-Grenoble, Département de Recherche Fondamentale sur la Matière Condensée, \\ SPSMS, 17 rue des Martyrs, F-38054 Grenoble Cedex 9, France
}

(Received 20 September 2001; published 11 April 2002)

\begin{abstract}
Thermodynamic (specific heat, reversible magnetization, tunneling spectroscopy) and transport measurements have been performed on high quality $(\mathrm{K}, \mathrm{Ba}) \mathrm{BiO}_{3}$ single crystals. The temperature dependence of the magnetic field $H_{C_{p}}$ corresponding to the onset of the specific heat anomaly presents a clear positive curvature. $H_{C_{p}}$ is significantly smaller than the field $H_{\Delta}$ for which the superconducting gap vanishes but is closely related to the irreversibility line deduced from transport data. Moreover, the temperature dependence of the reversible magnetization presents a strong deviation from the Ginzburg-Landau theory emphasizing the peculiar nature of the superconducting transition in this material.
\end{abstract}

DOI: $10.1103 /$ PhysRevLett.88.177201

PACS numbers: 74.25.Bt, 74.25.Dw, 74.60.Ec

The $H-T$ phase diagram of high $T_{c}$ superconducting oxides has been the focus of intense theoretical and experimental study during the past decade [1]. One of the most interesting phenomenon which has been observed is the existence of a melting line $T_{m}(H)$ above which the vortex lattice melts into a liquid of entangled lines [1]. This melting has very important consequences for the physics of vortices, since the free motion of the flux lines in the liquid state gives rise to a large dissipation and renders the system useless for applications. The presence of this liquid phase also hinders any direct determination of the upper critical field $H_{c 2}$ from standard magnetotransport data. Indeed, any resistive "critical" field $H_{R}(T)$, defined as the field for which the resistivity reaches some arbitrary value $R$ (e.g., $50 \%$ of the normal state resistivity), then presents a positive curvature [2]. Similarly, the presence of strong thermal fluctuations also complicates the determination of $H_{c 2}$ from thermodynamic measurements. These fluctuations lead to broad and smooth anomalies in specific heat measurements $\left(C_{p}\right)$. Thus the field $H_{C_{p}}$ (defined, for instance, by the inflection point of $C_{p} / T$ ) can present either a positive curvature in $\mathrm{Y}_{1} \mathrm{Ba}_{2} \mathrm{Cu}_{3} \mathrm{O}_{7-\delta}(\mathrm{YBCO})$ crystals [3], a linear dependence in $\mathrm{Tl}_{2} \mathrm{Ba}_{2} \mathrm{CuO}_{6}$ [4], or even almost no dependence with field in highly anisotropic systems such as $\mathrm{Bi}_{2} \mathrm{Sr}_{2} \mathrm{CaCu}_{2} \mathrm{O}_{8}$ [5] and $\mathrm{HgBa}_{2} \mathrm{Ca}_{2} \mathrm{Cu}_{3} \mathrm{O}_{8+\delta}$ [6]. It is still unclear whether this upper critical field still exists as a transition line or is just some smooth crossover between the vortex liquid and the normal state.

In order to shed light on this issue, we present thermodynamic (specific heat, magnetotunneling, reversible mag- netization) and transport measurements on high quality optimally doped $\left(\mathrm{K}_{x}, \mathrm{Ba}_{1-x}\right) \mathrm{BiO}_{3}$ single crystals $(x \sim 0.4)$. This compound is interesting since thermal fluctuations are expected to be small (the Ginzburg number $G_{i}$ is only $\sim 10^{-4}$ ) due to its isotropic structure, a $T_{c} \sim 31 \mathrm{~K}$, and a coherence length on the order of $30 \AA$. We show that the magnetic field $H_{C_{p}}$ presents a strong positive curvature and is smaller than the field $H_{\Delta}$ for which the superconducting gap vanishes, indicating a very peculiar nature for the thermodynamic superconducting transition in this system.

These measurements were performed on new particularly homogeneous single crystals presenting very sharp superconducting transitions in both transport $\left(\Delta T_{c} \sim 0.15 \mathrm{~K}\right)$ and ac susceptibility $\left(\Delta T_{c} \sim 0.2 \mathrm{~K}\right.$ for $\left.h_{a c}<0.01 \mathrm{G}\right)$ measurements. The specific heat was measured by an ac technique [7] which allows us to measure small samples (here a few $10^{-2} \mathrm{~mm}^{3}$ ) with high sensitivity (typically 1 part in $10^{4}$ ). Heat was supplied to the sample at a frequency $\omega$ on the order of a few $\mathrm{Hz}$ by a light emitting diode via an optical fiber. The induced temperature oscillations were measured by a chromelconstantan thermocouple that was calibrated in situ using a very pure silver single crystal as a reference. These measurements are only relative and were renormalized using the data from Ref. [8]. Figure 1 displays the temperature dependence of the specific heat at various magnetic fields up to $5 \mathrm{~T}$. The $7 \mathrm{~T}$ curve is equal to the normal state specific heat above $T=T_{c}(H=7 \mathrm{~T}) \sim 20 \mathrm{~K}$ and has been used as a base line in Fig. 1. 


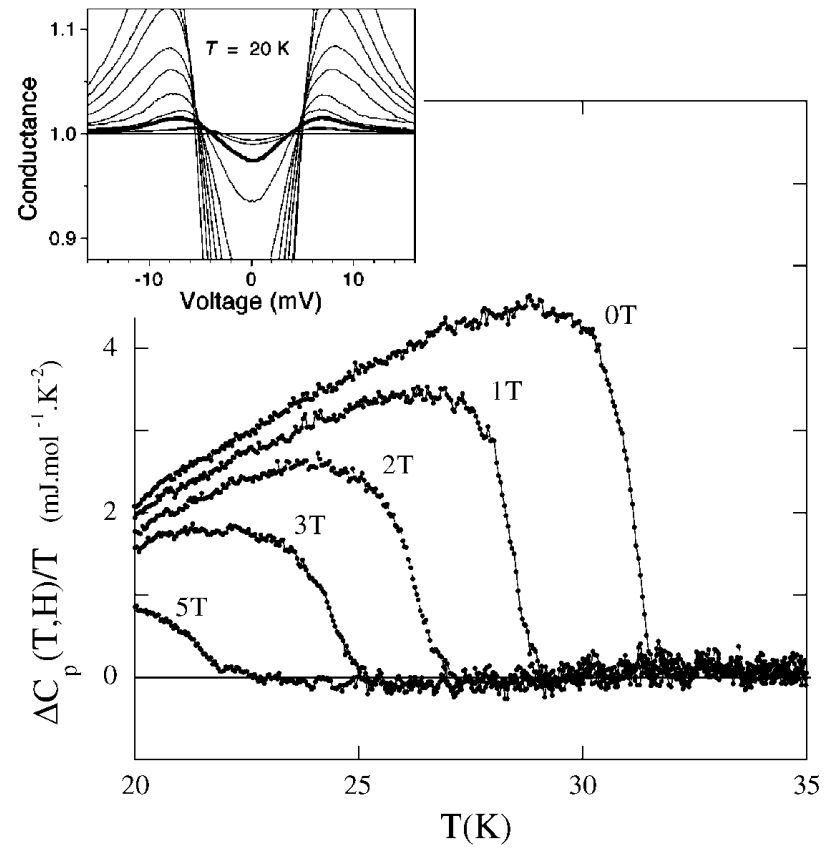

FIG. 1. Temperature dependence of the specific heat anomaly in a $(\mathrm{K}, \mathrm{Ba}) \mathrm{BiO}_{3}$ single crystal. $\Delta C_{p}=C_{p}(H, T)-C_{p}(H=$ $7 \mathrm{~T}, T)$. Inset: magnetic field dependence of the tunneling spectra at $T=20 \mathrm{~K}$ and $H(T)=0,1,2,3,4,5,6,7$ (bold curve), $8,9,10$.

The unusually high quality of our crystals is attested to by the narrow width of the transition, which is on the order of $1 \mathrm{~K}$ in zero magnetic field, and by the amplitude of the specific heat jump, $\Delta C_{p}\left(T_{c}\right) / T_{c} \sim 4-5 \mathrm{~mJ} \mathrm{~mol}^{-1} \mathrm{~K}^{-2}$. This ratio is $\sim 2$ and 5 times larger than that of Refs. [8] and [9], respectively, which are the only prior reports of a specific heat anomaly at $T_{c}$ in this system. This allowed us to study the magnetic field dependence of the specific heat anomaly in much greater detail. As shown in Fig. 1, this anomaly remains well defined in magnetic fields. The $T_{C_{p}}(H)$ curve corresponding to any characteristic point of the transition (e.g., the onset, midpoint, or maximum) presents a clear positive curvature. This can be seen in Fig. 2, where $T_{C_{p}}$ corresponds to the onset of the peak. The shaded area represents the temperature difference between the onset and the midpoint of the transition. A very similar upward curvature has been obtained in two other samples. A positive curvature was already obtained by transport [10] and magnetic measurements [11]. However, in nonclassical superconductors, there is an ambiguity in defining $H_{c 2}$ using those techniques.

Another striking feature of the specific heat anomaly is the rapid collapse of its height with field. Such behavior was previously observed in high $T_{c}$ materials [5,6] and is usually attributed to the presence of highly fielddependent thermal fluctuations. The observation of a similar collapse in $(\mathrm{K}, \mathrm{Ba}) \mathrm{BiO}_{3}$ remains a puzzling issue. Geometrical arguments based on entropy conservation imply that this reduction must be accompanied by a rapid, anomalous, increase of the specific heat at low

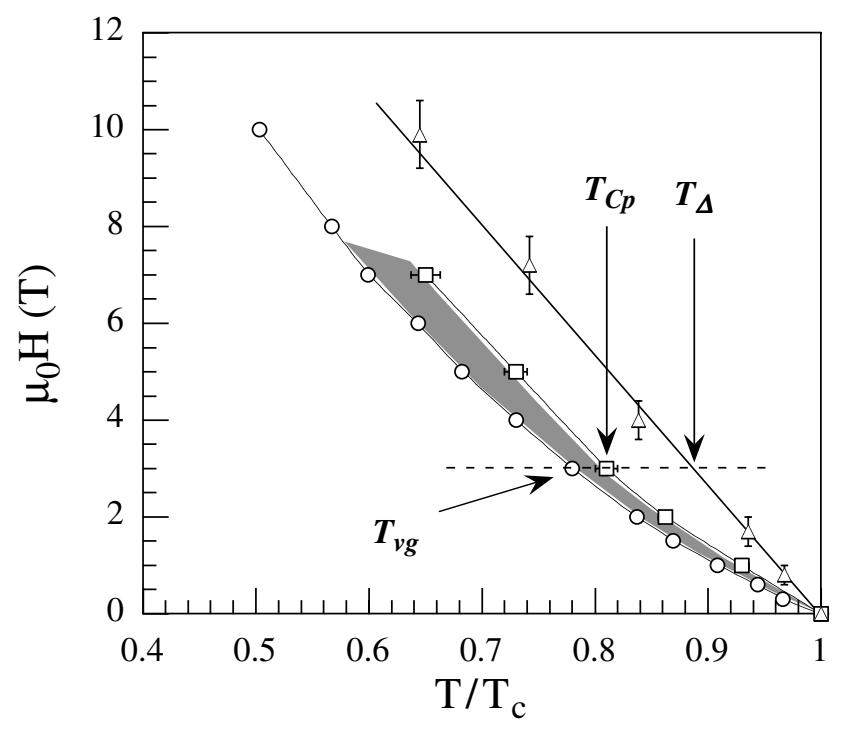

FIG. 2. $H-T$ phase diagram of the $(\mathrm{K}, \mathrm{Ba}) \mathrm{BiO}_{3}$ system. Squares $\left[H_{C_{p}}(T)\right]$ : onset of the specific heat anomaly (see Fig. 1; the shaded area represents the temperature difference between the onset and the midpoint of the anomaly), circles $H_{\mathrm{vg}}(T)$ : "vortex-glass transition" line deduced from transport measurements $(R \rightarrow 0)$; triangles: $H_{\Delta}(T)$ defined as the line for which the superconducting gap measured by tunneling spectroscopy vanishes. The different characteristic temperatures at $3 T$ (arrows) are as shown in Fig. 3.

temperature for fields much smaller than the upper critical field of $\sim 30 \mathrm{~T}$ deduced from transport data. The data from Ref. [8] also suggest such behavior.

The specific heat measurements are in striking contrast with our previous magnetotunneling [12] data which suggested that $H_{\Delta}$ presents a classical Werthamer-HelfangHohenberg (WHH) dependence. We have thus performed similar magnetotunneling measurements on the sample used in the specific heat experiments. The inset of Fig. 1 shows the evolution of the tunneling spectra near the normal state at $T=20 \mathrm{~K}$ with increasing magnetic fields. As shown by the bold curve, at $H=H_{C_{p}}(20 \mathrm{~K}) \sim 7 \mathrm{~T}$, a gap is still well developed and it closes at about $10 \mathrm{~T}$. As shown in Fig. 2, in agreement with our previous data, the curvature is much less pronounced for $H_{\Delta}$ and surprisingly $H_{C_{p}}<H_{\Delta}$. Note that, even though the difference between $T_{C_{p}}$ and $T_{\Delta}$ is quite large, the resistivity reaches about 95\% of its normal state value $\left(R_{N}\right)$ at $T_{C_{p}}$ and smoothly increases up to $\sim 100 \%$ for $T \sim T_{\Delta}$ (see Fig. 3). The specific heat anomaly thus defines a fundamental boundary below which the resistivity drops rapidly towards zero but superconductivity is only completely destroyed at $T=T_{\Delta}$ for which $R=R_{N}$ and the superconducting gap is completely suppressed. Note that the difference between $T_{\Delta}$ and $T_{C_{p}}$ is not due to sample homogeneities since heavy ion irradiation leads to an increase of $T_{C_{p}}$ which then tends towards $T_{\Delta}$ [13]. This difference could be due to the existence of a pseudogap but, in contrast to cuprates, this pseudogap would occur only in a nonzero magnetic field. 


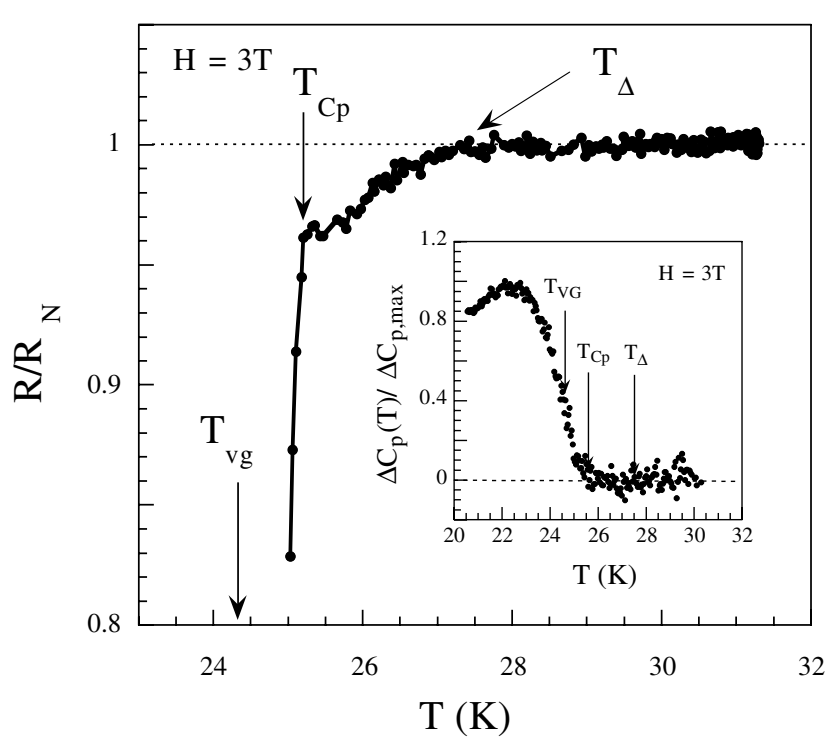

FIG. 3. Resistive transition at $H=3 \mathrm{~T}$. The onset of the specific heat anomaly (see Fig. 1) corresponds to the temperature below which the resistivity rapidly decreases. $R \rightarrow R_{N}$ for $T=T_{\Delta}$ (temperature for which the superconducting gap closes, and $R_{N}$ is the normal state resistivity). Inset, renormalized specific heat vs temperature. As shown, $T_{\mathrm{vg}}$ is close to the midpoint of the specific heat anomaly.

To complete this study of the thermodynamic properties, we performed reversible magnetization measurements $\left(M_{\text {rev }}\right)$ using a SQUID magnetometer. In the intermediate magnetic field range, $H_{c 1} \ll H \ll H_{c 2}$, the reversible magnetization of extreme type-II superconductors in the London model [14] is given by $M_{\mathrm{rev}}=-\frac{\alpha \Phi_{0}}{32 \pi \lambda^{2}} \ln \frac{\beta H_{\mathrm{c} 2}(T)}{H}$, where $\alpha \sim 1, \Phi_{0}$ is the flux quantum, $\lambda(T)$ is the magnetic penetration depth, $H_{c 2}(T)$ is the upper critical field, and $\beta \sim 0.37$ [15]. Thus, $\partial M_{\mathrm{rev}} / \partial \ln (H)$ is expected to be proportional to $1 / \lambda^{2}(T) \sim\left(1-T / T_{c}\right)$ close to $T_{c}$. $M_{\mathrm{rev}}$ presents a clear logarithmic dependence (see [16]), and the corresponding $\partial M_{\text {rev }} / \partial \ln (H)$ are shown in Fig. 4. However, as shown, strong deviations from the expected linear behavior are visible and $\partial M_{\text {rev }} / \partial \ln (H)$ can be described much better by a $\left(1-T / T_{c}\right)^{1.5}$ law (solid line, the dotted line is a WHH dependence for $1 / \lambda^{2}$ ). A similar deviation is usually observed in high $T_{c}$ cuprates [17] and has been attributed to the presence of strong fluctuations. It was originally suggested by Nelson et al. [18] that elastic distortions of the vortex lattice (in the presence of strong thermal fluctuations) may lead to a significant contribution to the entropy of the system. However, the corresponding contribution to $\partial M_{\mathrm{rev}} / \partial \ln (H)$ is expected to be of the order of $k_{B} T / \Phi_{0} \xi$ [19] (where $k_{B}$ is the Boltzmann constant), which is about 1 order of magnitude smaller than the observed deviation in $(\mathrm{K}, \mathrm{Ba}) \mathrm{BiO}_{3}$ [20].

Our specific heat and magnetization measurements give new insight into the magnetotransport data. Those data are well described by the vortex-glass scaling formalism [10], suggesting that the vortex solid melts into a liquid at high temperature through a second order phase tran-

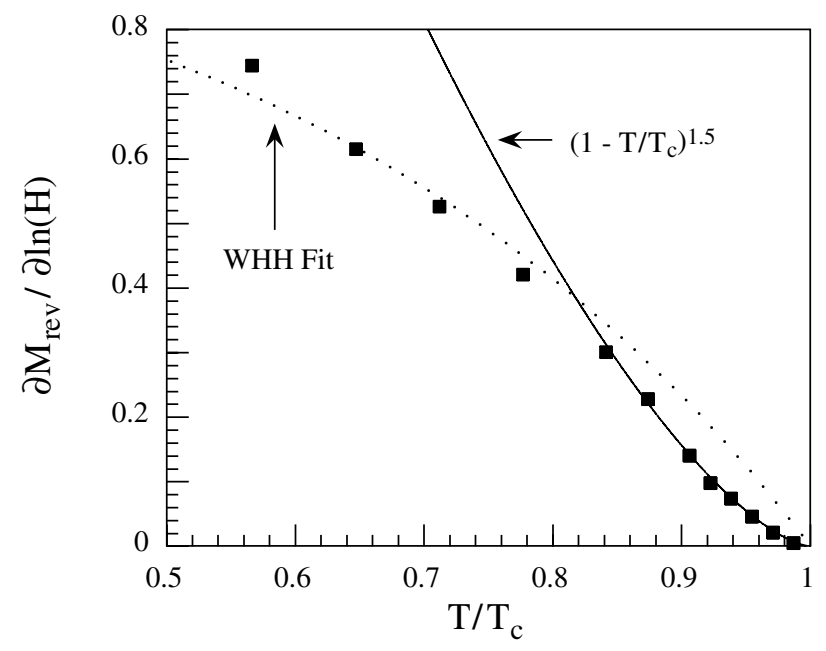

FIG. 4. Temperature dependence of $\partial M_{\text {rev }} / \partial \ln (H)$ in a $(\mathrm{K}, \mathrm{Ba}) \mathrm{BiO}_{3}$ single crystal. The dotted line is a WHH fit for the temperature dependence of $1 / \lambda^{2}$ (see text for details). The solid line is a $\left(1-T / T_{c}\right)^{1.5}$ fit to the data.

sition. The physical image emerging from those measurements was then that of a liquid phase existing for $H_{\text {vg }}<H<H_{\Delta} \sim H_{c 2}$ (the vortex-glass transition field $H_{\mathrm{vg}}$ is defined as the field for which $R \rightarrow 0$ ). However, as shown in Fig. $2, H_{\mathrm{vg}}$ and $H_{C_{p}}$ present a very similar temperature dependence emphasizing the close relationship between those two lines. As shown in the inset of Fig. 3, the $R=0$ criterion is close to the midpoint of the $C_{p}$ transition. This then suggests two possibilities: either (i) the positive curvature in $H_{\mathrm{vg}}$ is not due to the melting of the vortex solid but is an intrinsic property of the $H_{c 2}$ line $\left(=H_{C_{p}}\right)$ or (ii) the specific heat anomaly is not related to $H_{c 2}\left(=H_{\Delta}\right)$ but instead marks a transition in the vortex state. Many theoretical models have been developed in order to explain a "possible" upward curvature of the upper critical field in cuprates (including a spin-charge separation [21] or very strong coupling [22]) but none of those models can be applied to the $(\mathrm{K}, \mathrm{Ba}) \mathrm{BiO}_{3}$ system. A positive curvature has also been predicted by Ovchinnikov and Kresin [23] in the presence of inhomogeneities and/or magnetic impurities. However, in this case this curvature is expected to appear only at low temperature (i.e., when the superconducting coherence length becomes smaller or on the order of the size of the impurity) and $H_{c 2}$ is still expected to vary linearly with $T$ close to $T_{c}$. Finally, the condensation of charged bosons in the magnetic field is also expected to lead to a positive curvature [24]. But one has then to take into account the fact that we do not observe any feature in the tunneling spectra above $T_{C_{p}}$ in zero magnetic field. On the other hand, a second scenario is that $H_{c 2} \sim H_{\Delta}$ and that the specific heat anomaly marks a transition within the vortex state. For instance, specific heat anomalies, both peaks and/or steps, have been observed for transitions in the vortex state in YBCO $[25,26]$. However, even though the amplitude of $\Delta C_{p}$ per unit volume 
measured here is similar to the one associated with vortex melting in YBCO, its shape is quite different and looks much like a classical superconducting transition.

The $(\mathrm{K}, \mathrm{Ba}) \mathrm{BiO}_{3}$ system thus presents very unusual behavior. Indeed, any characteristic field deduced either from specific heat or from transport measurements presents a strong positive curvature; a structure in the density of states is visible well above the specific heat anomaly and strong deviations from the standard Ginzburg-Landau theory are observed in the reversible magnetization. This behavior is reminiscent of high $T_{c}$ oxides, where strong fluctuations occur. In $(\mathrm{K}, \mathrm{Ba}) \mathrm{BiO}_{3}$, thermal fluctuations are expected to play only a minor role, but the superconducting transition occurs in the vicinity of a metal-insulator transition. The very small carrier density might thus lead to the presence of large quantum fluctuations [27,28]. Further studies are needed to fully understand the superconducting transition in this remarkable system.

We thank L. Paulius for interesting discussions and N.E. Phillips and R. A. Fischer for sending us their specific heat data on $(\mathrm{K}, \mathrm{Ba}) \mathrm{BiO}_{3}$.

[1] G. Blatter, M. V. Feigel'man, V. B. Geshkenbein, A. I. Larkin, and V. M. Vinokur, Rev. Mod. Phys. 66, 1125 (1994).

[2] See, for instance, I. W. Sumarlin et al., Phys. Rev. Lett. 68, 2228 (1992); A. P. Mackenzie et al., Phys. Rev. Lett. 71, 1238 (1993); M. S. Osofski et al., Phys. Rev. Lett. 71, 2315 (1993).

[3] M. Roulin, A. Junod, and E. Walker, Physica (Amsterdam) 296C, 137 (1998).

[4] A. Carrington, A. P. Mackenzie, and A. Tyler, Phys. Rev. B 54, R3788 (1996).

[5] A. Junod, A. Erb, and C. Renner, Physica (Amsterdam) 317C-318C, 333 (1999).

[6] C. Marcenat et al., in Coherence in High Temperature Superconductors (World Scientific, Singapore, 1996); A. Carrington et al., Phys. Rev. B 55, R8674 (1997).

[7] P. F. Sullivan and G. Seidel, Phys. Rev. 173, 679 (1968).

[8] B. F. Woodfield, D. A. Wright, R. A. Fisher, N. E. Philips, and H. Y. Tang, Phys. Rev. Lett. 83, 4622 (1999).

[9] J. E. Graebner, L. F. Schneemeyer, and J. K. Thomas, Phys. Rev. B 39, 9682 (1989).

[10] T. Klein, A. Conde-Gallardo, J. Marcus, P. Szabo, P. Samuely, A. G. M. Jansen, and C. Escribe-Filippini, Phys. Rev. B 58, 12411 (1998).
[11] V.F. Gantmakher et al., Phys. Rev. B 54, 6133 (1996); D. Hall et al., Philos. Mag. B 80, 61 (2000).

[12] P. Samuely, P. Szabo, T. Klein, A. G. M. Jansen, J. Marcus, C. Escribe-Filippini, and P. Wyder, Europhys. Lett. 41, 207 (1998).

[13] C. Marcenat et al. (to be published).

[14] P. De Gennes, in Superconductivity of Metals and Alloys (Addison-Wesley, New York, 1989); M. Tinkham, in Introduction to Superconductivity (McGraw-Hill, New York, 1996); A. A. Abrikosov, in Fundamentals of the Theory of Metals (North-Holland, New York, 1988).

[15] This model was later improved by Z. Hao and J. R. Clem [Phys. Rev. Lett. 67, 2371 (1991)], taking into account the contribution of the vortex core to the free energy density. Despite this nonlogarithmic contribution to the magnetization, it has been shown that $M_{\text {rev }}$ remains approximatively linear with $\ln H$ on a large magnetic field range with $\alpha \sim 0.77$ and $\beta \sim 1.44$.

[16] I. Joumard, T. Klein, A. Conde-Gallardo, J. Marcus, and A. Sulpice, Physica (Amsterdam) 341C-348C, 1067 (2000).

[17] See, for instance, P. H. Kes et al., Phys. Rev. Lett. 67, 2383 (1991); U. Welp et al., Phys. Rev. Lett. 67, 3180 (1991).

[18] D. R. Nelson and H.S. Seung, Phys. Rev. B 39, 9153 (1989).

[19] L. N. Bulaevskii et al., Phys. Rev. Lett. 68, 3773 (1992). In highly anisotropic samples the coherence length $\xi$ should be replaced by the interlayer spacing $s$.

[20] The main difference between $(\mathrm{K}, \mathrm{Ba}) \mathrm{BiO}_{3}$ and cuprates is that $\partial M_{\text {rev }} / \partial \ln (H)$ remains positive on the entire temperature range in $(\mathrm{K}, \mathrm{Ba}) \mathrm{BiO}_{3}$.

[21] R. G. Dias and J. M. Wheatley, Phys. Rev. B 50, R13 887 (1994).

[22] F. Marsiglio and J.P. Carbotte, Phys. Rev. B 36, 3633 (1987).

[23] Y. N. Ovchinnikov and V.Z. Kresin, Phys. Rev. B 54, 1251 (1996).

[24] A. S. Alexandrov, Phys. Rev. B 48, 10571 (1993).

[25] A. Schilling et al., Nature (London) 382, 791 (1996); M. Roulin, Science 273, 1210 (1996).

[26] F. Bouquet, C. Marcenat, E. Steep, R. Calemczuk, W. K. Kwok, U. Welp, G. W. Crabtree, R. A. Fisher, N.E. Phillips, and A. Schiling, Nature (London) 411, 448 (2001).

[27] G. Blatter and B. I. Ivlev, Phys. Rev. B 50, 10272 (1994).

[28] V. J. Emery and S. A. Kivelson, Nature (London) 374, 434 (1995); ibid., Phys. Rev. Lett. 74, 3253 (1995). 